\title{
A Case of Successful Management of GuillainBarre Syndrome in Pregnancy
}

\author{
Debasis Pradhan, ${ }^{1}$ Samarjit Dey, ${ }^{2}$ Prithwis Bhattacharyyaa ${ }^{2}$ \\ 'Junior Consultant, Columbia Asia hospital, Hebbal, Bengaluru, India, ${ }^{2}$ Department of Anesthesiology \& Critical Care, \\ NEIGRIHMS, Shillong, India
}

\section{ABSTRACT}

Guillain-Barre' syndrome (GBS) complicating pregnancy is a rare event which carries a high maternal risk. We presented a case of 23 years old pregnant lady diagnosed with GBS in third trimester who deteriorated after termination of delivery of the new born. Despite the availability of intravenous immunoglobulin and plasmapheresis and complications like ventilator associated pneumonia, tracheostomy, autonomic instability, patient was successfully managed due to a meticulous multidisciplinary involvement in our intensive care unit.

Keywords: pregnancy; thromboprophylaxis; tracheostomy; ventilator associated pneumonia.

\section{INTRODUCTION}

Guillain-Barre syndrome (GBS) is an acute inflammatory demyelinating polyneuropathy of autoimmune etiology with incidence ranging from 0.75 to two cases per 100,000 people a year. The disease occurrence in the last trimester of pregnancy increases maternal risks of respiratory complications, needing ventilatory support in $34.5 \%$ of the cases and increasing risk of prematurity. ${ }^{1}$

Here we are reporting successful management of a case of Guillain-Barre syndrome in pregnancy, which required prolonged mechanical ventilation. Her total stay in ICU was 12 weeks and total mechanical ventilation period was eight weeks.

\section{CASE REPORT}

A 23-year-old lady in her third pregnancy, at 36 weeks of gestation presented to emergency Department with the complaints of difficulty in breathing and swallowing for past two days and weakness in all four limbs for past 4 months without fecal and urinary incontinence. She had gradual onset of weakness in all four limbs which was preceded by fever without any history of dysentery. Previous medical and obstetric histories were not significant. Neurological examination revealed normal higher function, decreased power in all four limbs (Upper limb-3/5, Lower limb 2/5), and bilateral absence of deep tendon reflexes, mute plantar response and no sensory loss. Size of the uterus was corresponding to the obstetric history. Fetal heart rate was $130-140$ per minute and cardiotocographic monitoring was started. Then patient was transferred to ICU and both maternal and fetal monitoring was continued.

Investigations included blood biochemistry, X-ray chest, ECG, CSF analysis. CSF report showed albumincytological dissociation. From the above history, clinical findings and investigation reports, probable diagnosis of GBS was made with Neurologists and Obstetrician consultation.

On day two, normal vaginal delivery was conducted by obstetric team in ICU and the new born was taken care by Pediatric attending physician.

On day three, (48 hrs post delivery) she had to be intubated and put on ventilator (Galileo Gold Ventilator from Hamilton Medical System, Switzerland) because of severe respiratory distress and progressive weakness in all four limbs (Both upper and lower limb 0/5). Closed suction system (Ty care, Tyco Healthcare, UK) was used. Enteral nutrition was initiated through nasoduodenal

Correspondence: Dr. Debasis Pradhan, Columbia Asia hospital, Hebbal, Bengaluru, India. Email: dmkcg85@gmail.com, Phone : +91. 8014770422 
tube (Freka Tube, Fresenius Kabi, Germany) as per caloric requirements with multivitamins and trace elements. Along with air mattress, frequent position change, limb and chest physiotherapy was initiated and for DVT prophylaxis sequential compression device pump (SCD express compression system, Tyco healthcare, Germany) and LMWH (Enoxaparin, $40 \mathrm{mg}$ subcutaneously, Once a day) were started.

On day eight, Patient developed fever and tachycardia with gradually increasing TLC and copious Endotracheal (ET) secretions with infiltrations in the Chest X-ray. Chest infection was suspected and injection Meropenem $1 \mathrm{gm}$ TDS was started, after sending ET secretion for gram stain and culture sensitivity.

On day 11, tracheostomy was done. Acinetobacterbaumanii and Klebsiella pneumonia ( $>10^{5}$ Colony Forming Units), which were sensitive to meropenem, were isolated from ET secretion. Proper hand hygiene was maintained. Psychological evaluation of the patient in presence of husband was done. Husband was involved in physiotherapy and mobilisation.

On day 19 , with mute deep tendon reflex motor activity gradually started improving (Power $2 / 5$ in upper limb and $1 / 5$ in lower limb).

On day 20, with the onset of spontaneous breathing, deep breathing exercise and spontaneous breathing trials were started.

On day 28, central venous catheter was removed and all medications were administered orally.

On day 40 , power improved to $3 / 5$ in all four limbs. Foley's catheter clamping and declamping was started. Abdominal and diaphragmatic muscle activities were weak with ineffective cough. Spontaneous breathing with pressure support alternating with SIMV was used. All supportive therapies were continued.

On day 50, T-piece trial was initiated, but failed because of failure to clear out secretion and patient apprehension.

On day 53 , patient was counselled by psychiatrist in presence of her husband and T-piece trial alternating with spontaneous breathing with pressure support was started. Patient tolerated T-piece trial. There were no signs of infection and all therapies continued.

On day 65, patient started taking orally and was maintaining $\mathrm{SpO}_{2}$ on room air.

On day 73, mobilization was started and patient was repeatedly motivated to cough voluntarily. Nebulization, chest and limb physiotherapy were continued.
On day 75 , power in all four limbs was found to be $4 / 5$ and fenestrated tracheostomy tube was placed.

On day 79, tracheostomy tube was closed externally and patient was maintaining $\mathrm{SpO}_{2}$ without oxygen supplementation. Following successful decanulation on $82^{\text {nd }}$ day patient was shifted to ward on $84^{\text {th }}$ day.

\section{DISCUSSION}

GBS is suspected when a patient presents with progressive motor weakness and areflexia, the only criteria that must be present for the diagnosis. ${ }^{2}$ Commonly, patients would have a history of upper respiratory tract infection $(40 \%)$ or gastroenteritis $(20 \%)$ within four weeks prior to onset of disease. ${ }^{3}$ However, the clinical spectrum of GBS is wide. Other clinical features include sensory symptoms, cranial nerve involvement (most commonly facial palsy), autonomic dysfunction causing pulse and blood pressure changes, and respiratory failure a major cause of morbidity and mortality. ${ }^{2}$ Laboratory and electrophysiological investigations can be useful in diagnosing GBS in both nonpregnant and pregnant patients, but neither of them is specific for the disease and they may not always be present. Albumino-cytologic dissociation in cerebrospinal fluid with elevated protein content and a normal mononuclear leukocyte count $\left(10 / \mathrm{mm}^{3}\right)$ are common findings and are strongly suggestive of GBS. ${ }^{3}$

Among all known infectious agents, Campylobacter jejuni has been recognized recently as the most common pathogen associated with GBS in the nonpregnant population. Cytomegalovirus (CMV) has been found to be the second most common infectious agent associated with GBS in the nonpregnant population. ${ }^{4}$

Randomized controlled trials showed that plasmapheresis is effective in treatment of GBS compared to supportive management with shorter duration of mechanical ventilation and reduced time to full ambulation. Improved outcome was especially observed in those with plasmapheresis started within two weeks of symptom onset. Intavenous Immunoglobin (IVIG) and plasmapheresis were found to be equally effective in treatment of GBS GBS on its own is therefore not an indication for termination of pregnancy. ${ }^{3}$

Specific treatments for GBS have been discussed as IVIG or plasmapheresis. Attention should be paid to the identification and treatment of infective complications, prevention of venous thromboembolism, pain management, and the management of psychological distress resulting from the disease and anxiety. The incidence of pulmonary embolism in nonpregnant GBS was reported to be between one and $13 \% .{ }^{3}$ Prophylactic anticoagulation in the immobilized GBS patient is considered a standard management in most units. ${ }^{5}$ 
Nosocomial infection is an important complication of GBS patients. As many as $25 \%$ of GBS patients acquired pneumonia and $30 \%$ suffered from urinary tract infections. ${ }^{6}$ Because these infections tend to be more severe in pregnant women, early identification and treatment are desirable. ${ }^{3}$

Despite advances in GBS treatment and critical care standards, in developing countries where intravenous immunoglobulin and plasmapheresis are not feasible, preventive measures towards maternal hemodynamics, respiratory, infectious and thrombotic complications along with psychological, nutritional, and physiotherapeutic supports will contribute towards better patient outcome.

\section{REFERENCES}

1. Campanharo FF, Santana EF, Sarmento SG, Mattar R, Sun SY, Moron AF. Guillain-Barré Syndrome after H1N1 Shot in Pregnancy: Maternal and Fetal Care in the Third TrimesterCase Report. Case Reports in Obstetrics and Gynecology. 2012 Nov 14;2012. doi:10.1155/2012/323625.

2. Asbury AK, Cornblath DR. Assessment of current diagnostic criteria for Guillain-Barre' syndrome. Ann Neurol. 1990;27 Suppl:S21-4.

3. Chan LYS, Tsui MHY, Leung TN. Guillain-Barré syndrome in pregnancy. Acta Obstetriciaet Gynecologica Scandinavica. 2004;83(4):319-25.

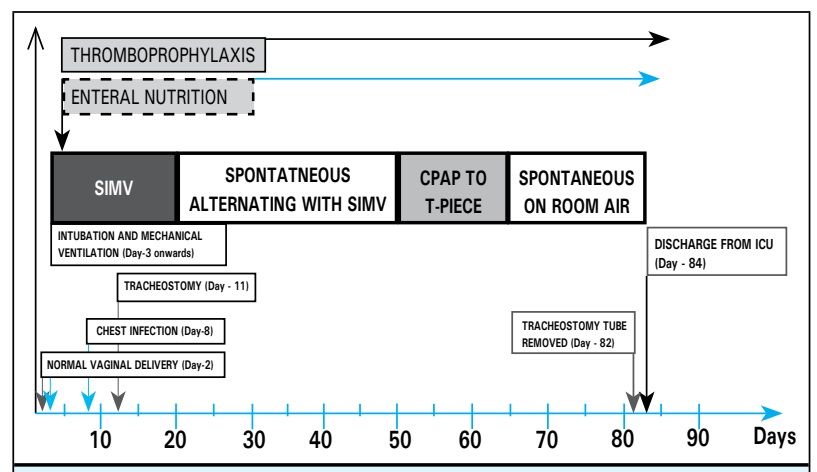

Figure 1. Major events during the management of the patient.

\section{ACKNOWLEDGEMENTS}

We thank all the nursing staffs of ICU NEIGRIHMS.
4. Jacobs BC, Rothbarth PH, van der Meche FG, Herbink P, Schmitz PI, de Klerk MA et al. The spectrum of antecedent infections in GuillainBarre' syndrome: a case-control study. Neurology. 1998 Oct; 51:1110-5.

5. Gaber TA, Kirker SG, Jenner JR. Current practice of prophylactic anticoagulation in Guillain-Barre' syndrome. ClinRehabil. 2002 Mar;16(2):190-3.

6. Ropper AH. The Guillain-Barre' syndrome. N Engl J Med. 1992 Apr 23;326(17):1130-6. 